\title{
Optimization of Sight Clearance Area Design at Uncontrolled Intersection
}

\author{
Wen Shao, Xuedong Yan, Yunwei Li \\ Beijing Jiaotong University, China
}

Keywords: Intersection sight triangle, significant impact on the driver's available sight.

\begin{abstract}
Since the lack of traffic control measures, it is necessary to provide the sufficient intersection sight distance to ensure the safety of driving at uncontrolled intersections. At present, the domestic and foreign design standards and specifications concentrate more on the dimensions of square edges and the internal clearance of the intersection sight triangle, and there is no reference to the layout of the outer area of the sight triangles. In fact, the location of the peripheral obstacles may have a significant impact on the driver's available sight and the information obtaining about the intersecting roads. Therefore, it's necessary to discuss the quantitative relation between the obstacle position and driving safety and find the optimal placement position of the obstacle. In this way a larger available range of sight and a higher safety of the vehicle can be provided to drivers during travelling.
\end{abstract}

\section{Introduction}

Statistical data show that in China, accidents occurring at intersections account for 20 to 30 percent of the total number of road traffic accidents in urban areas [1]. And the ratio is about 35 percent in the United States [2], while in Germany the number of road traffic accidents at intersections is 60 to 85 percent [3]. It can be seen that the traffic safety of intersections plays an important role in the safety of the whole road network.

There are many factors affecting the traffic safety at intersections, and sight distance is an important factor among them. According to statistics, Hanna et al. pointed out that at intersections with poor sight distance the accident rate of millions of cars was 1.33 , and 1.13 at intersections with good sight distance condition [4]. Bernd et al. counted 450 crashes at intersections in Germany, of which 16 percent were caused by insufficient sight distance [5]. Because of the lack of traffic control measures, it is necessary to provide sufficient sight distance for drivers to ensure the safety of driving. Good sight distance conditions can enable vehicles to detect potential conflict vehicles in time, and also enable their vehicles to be detected by the potential vehicle in time to reduce the possibility of collision [6].

At present, most domestic and foreign design specifications are concerned with the design of the dimensions of the sight triangle. Design standards only stipulated that the interior of the sight triangle must remain clear, but there is no mention of the position of the perimeter of the sight triangle. Meanwhile, scholars' studies did not focus on that peripheral obstacle position might affect the driver's available sight.

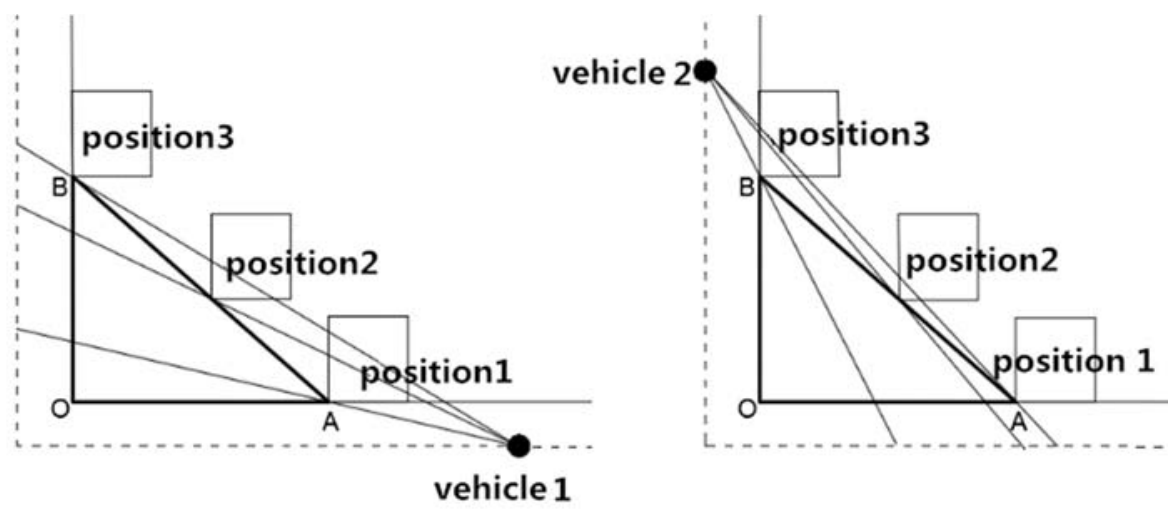

Figure 1. Different range of sight in different position of the obstacle 
Therefore, in this paper a sight clearance area geometric model is set up, to explore the quantitative relationship between the position of obstacles and driver's available sight distance and find the optimal placement of the obstacles to improve the driving safety.

\section{Effect of The Location of Obstacles}

When the drivers' range of sight increases, more potential conflict vehicles can be seen in time, so that drivers can take actions in advance to avoid collision and improve the safety of driving. As shown in figure 1, the triangle $\mathrm{OAB}$ is the sight triangle clearance area. There are three kinds of positions of obstacles beside the hypotenuse of the sight triangle. Position 1, 2, and 3 can provide drivers different range of sight.

\section{Optimal Tangent Form}

According to the design standards, the interior area of the sight triangle must be clear to ensure that the driver's sight would not be blocked, and there can be obstacles outside. Thus there are two kinds of tangent forms in this case between the obstacle and the hypotenuse of sight triangle: tangent to one side and tangent to one point.

\subsection{The Obstacle and the Hypotenuse Tangent to One Side}

As shown in figure 2(a), the obstacle and the hypotenuse tangent to the side $\mathrm{MN}$, when the vehicle from the direction $\mathrm{x}$ observes the potential vehicle from the direction $\mathrm{y}$, the point $\mathrm{M}$ will block the driver's sight; when the vehicle from the direction y observes the potential vehicle from the direction $\mathrm{x}$, the point $\mathrm{N}$ will block the driver's sight too.

\subsection{The Obstacle and the Hypotenuse Tangent to One Point}

As shown in figure 2(b), the obstacle and the hypotenuse tangent to a point $\mathrm{P}$, when the vehicle from the direction $\mathrm{x}$ observes the potential vehicle from the direction $\mathrm{y}$ and when the vehicle from the direction $\mathrm{y}$ observes the potential vehicle from the direction $\mathrm{x}$, the point that will block drivers' sight is the point $\mathrm{P}$.

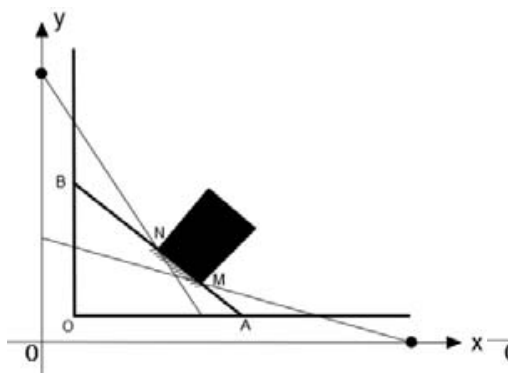

(a)

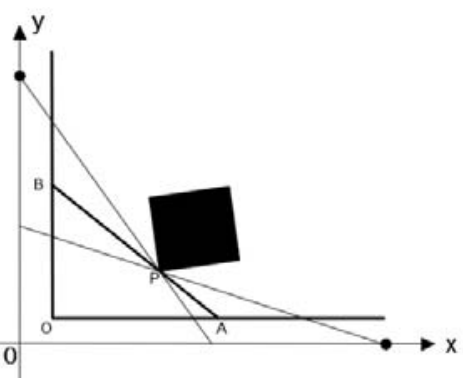

(b)

Figure 2. Two tangential forms between obstacles and hypotenuses of sight triangle

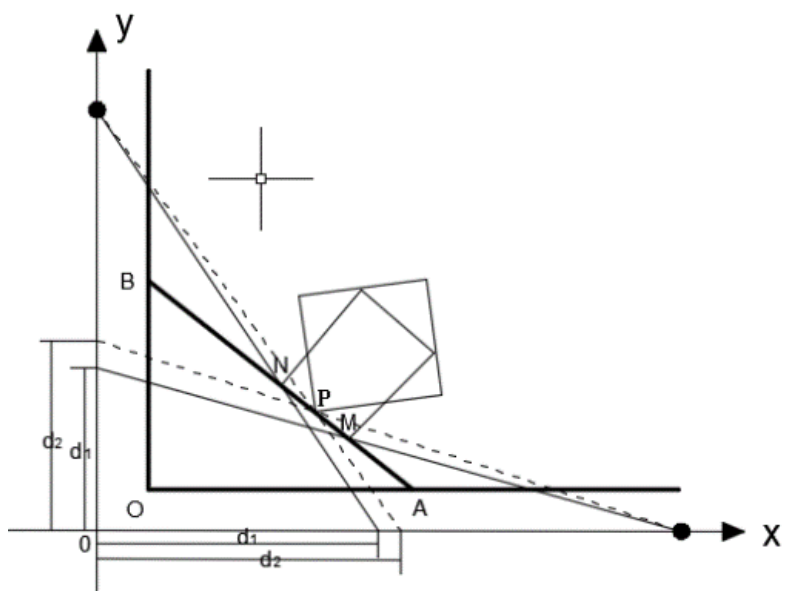

Figure 3. The optimal tangential form 


\subsection{The Comparison of Two Tangent Forms}

From the perspective of driver's available range of sight, as shown in figure 3, we can change the tangent side to the tangent point. When the slope of the line between two conflict vehicles is greater than the slope of the hypotenuse of the sight triangle, the sight blocking point will move from the point $\mathrm{N}$ to the point $\mathrm{P}$, and drivers' available range of sight will change from $\mathrm{d} 1 \mathrm{to} \mathrm{d} 2$. On the contrary, the sight blocking point will move from the point $\mathrm{M}$ to the point $\mathrm{P}$, and drivers' available range of sight will change from $\mathrm{d} 1$ to $\mathrm{d} 2$. Thus drivers could see more potential conflict vehicles and we can get the conclusion that the optimal tangential form is tangent to one point.

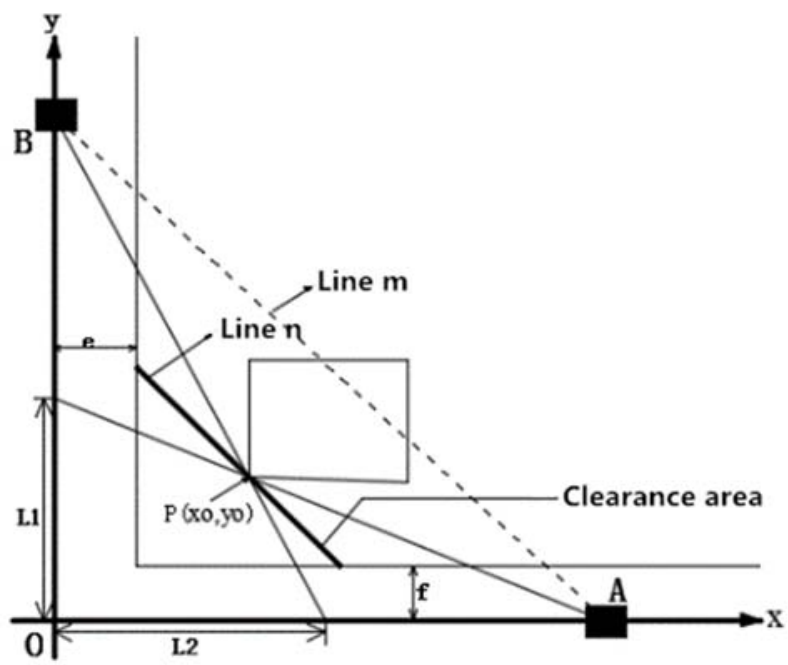

Figure.4 Calculation of the optimal obstacle location

\section{The Optimal Tangent Point Position}

As shown in Figure 1 earlier, for the vehicle 1 traveling from east to west, as the obstacle gradually moves from position 1 to position 3, the tangent point gradually moves from A to B, and the driver's range of sight can be gradually increased; for the vehicle 1 traveling from north to south, as the obstacle gradually moves from position 1 to position 3 , the tangent point gradually moves from A to $\mathrm{B}$, and the driver's range of sight can be gradually decreased.

Therefore, it is necessary to find a point on the hypotenuse $\mathrm{AB}$ between the tangent points $\mathrm{A}$ and $\mathrm{B}$ corresponding to the position 1 and the position 3 so that the sum of the range of sight obtained by the vehicles 1 and 2 is the maximum when the tangent point is located at this point. In that way we can find the optimal tangency point position to place the obstacle.

As shown in Figure 4, a geometric model of driver's sight is established in the Cartesian coordinate system to find the optimal tangent point position to place the obstacle.

If the slope of line $\mathrm{m}$ and $\mathrm{n}$ are the same, then the equation of the line is set as:

Line $\mathrm{n}$ and line $\mathrm{m}$ :

$$
\begin{aligned}
& y=k x+b \\
& y=k x+d
\end{aligned}
$$

The equation of line $\mathrm{AP}$ and line $\mathrm{BP}$ can be obtained from $\mathrm{A}, \mathrm{P}$ and $\mathrm{B}, \mathrm{P}$ respectively.

Line AP and line BP is set as follows:

$$
\begin{gathered}
\mathrm{y}=(\mathrm{kx} 0+\mathrm{b}) /(\mathrm{x} 0+\mathrm{d} / \mathrm{k})+(\mathrm{dkx} 0+\mathrm{bd}) /(\mathrm{kx} 0+\mathrm{d}) \\
\mathrm{y}=\mathrm{kx}+(\mathrm{b}-\mathrm{d}) / \mathrm{x} 0+\mathrm{d}
\end{gathered}
$$

Lo is the objective function, which represents the sum of the sight of two vehicles:

$$
\mathrm{Lo}=\mathrm{L} 1+\mathrm{L} 2=(-\mathrm{dx} 0+\mathrm{bc}) /(\mathrm{c}-\mathrm{x} 0)-(\mathrm{dx} 0) /(\mathrm{kx} 0+\mathrm{b}-\mathrm{d})
$$

In the formula, the independent variable is $\mathrm{x}$, which is, the abscissa of point $\mathrm{P}$, the value range is set as follows:

$$
\mathrm{e} \leq \mathrm{x} 0 \leq(\mathrm{f}-\mathrm{b}) / \mathrm{k}
$$

In the above formula, $\mathrm{k}, \mathrm{b}, \mathrm{c}$ and $\mathrm{d}$ are constants. Taking $\mathrm{c}=\mathrm{d}=100, \mathrm{~b}=50$ and $\mathrm{e}=\mathrm{f}=3$, then $\mathrm{k}$ $=-1$. At this time, when $x=25$, the derivative $y^{\prime}=0$ and the objective function $y$ obtains the maximum 
value. That is, when the tangent point on the hypotenuse has an abscissa of 25 , the total range of sight obtained by both parties can be maximized.

The above calculation results of the sight distance geometry model show that when obstacles are distributed along the periphery of the sight triangle hypotenuse, there is a certain location makes obstacles placed here can provide a maximum range of the sight to the drivers. In this way drivers can see more potential conflict vehicles and take actions to avoid collision in time.

\section{Conclusion}

This paper proposes that at uncontrolled intersections, when two potential conflict vehicles are approaching each other, the shape of the clearance area of the sight triangles will have a significant impact on the driver's sight, and proposes that the different placement of obstructions at the periphery of the sight triangles will determine the range of sight that the driver can obtain. By establishing the geometric model of the sight distance clearance area at uncontrolled intersections considering the placement of obstacles, the advantages and disadvantages of different tangent forms between the position of obstructions and the hypotenuse of sight triangles are discussed.

The analysis results show that when there are obstacles on the periphery of the sight triangle, drivers can get a greater range of sight if the obstacles and the hypotenuse tangent to a point rather than tangent to a side. Moreover, there is an optimal tangent point on the hypotenuse of the sight triangle, and if the obstruction is tangent to the hypotenuse of the sight triangle at this optimal tangent point, both two potential conflict drivers can get the maximum range of sight when observing each other.

\section{References}

[1]. Anwen Wen, Hui Hu, Xiang Li. The Relationship of Intersections and Traffic Safety. [J]. Traffic Standardization, 2010, 13: 108-111.

[2]. Lord, D., Schalkwyk, I.V., Staplin, L., Chrysler, S. Reducing Older Driver Injuries at Intersection Using More Accommodating Design Practices [C], Accident Analysis and Prevention Texas Transportation Institute, 2005.

[3]. Yan Wu, Study on Highway Intersections Safety [D].Xian: Chang' a university, 2008.

[4]. Kreston. Haydn. Traffic Conflict Technology [M]. Su Zhang, Translate. Chengdu: Southwest Jiaotong University Press, 1994.

[5]. Yongyuan Qin. Principle of Kalmar filter and Integrated Navigation [M]. Xi 'an Industrial University Press,2004:1.3,33-40

[6]. AASHTO: A Policy on Geometric Design of Highways and Streets [M]. Washington, D.C., TRB, 2011, 88-173. 